\title{
Genetic Algorithm for Multiple Bus Line Coordination on Urban Arterial
}

\author{
Zhen Yang, ${ }^{1,2}$ Wei Wang, ${ }^{1,2}$ Shuyan Chen,,2 Haoyang Ding, ${ }^{1,2}$ and Xiaowei Li ${ }^{1}$ \\ ${ }^{1}$ Jiangsu Key Laboratory of Urban ITS, Southeast University, 2 Si Pai Lou, Nanjing 210096, China \\ ${ }^{2}$ Jiangsu Province Collaborative Innovation Center of Modern Urban Traffic Technologies, 2 Si Pai Lou, Nanjing 210096, China
}

Correspondence should be addressed to Zhen Yang; yzyz3770@163.com

Received 24 September 2014; Revised 13 December 2014; Accepted 18 December 2014

Academic Editor: Yongjun Shen

Copyright (C) 2015 Zhen Yang et al. This is an open access article distributed under the Creative Commons Attribution License, which permits unrestricted use, distribution, and reproduction in any medium, provided the original work is properly cited.

Bus travel time on road section is defined and analyzed with the effect of multiple bus lines. An analytical model is formulated to calculate the total red time a bus encounters when travelling along the arterial. Genetic algorithm is used to optimize the offset scheme of traffic signals to minimize the total red time that all bus lines encounter in two directions of the arterial. The model and algorithm are applied to the major part of Zhongshan North Street in the city of Nanjing. The results show that the methods in this paper can reduce total red time of all the bus lines by $31.9 \%$ on the object arterial and thus improve the traffic efficiency of the whole arterial and promote public transport priority.

\section{Introduction}

In recent years, bus priority strategy as a possible way to solve urban traffic problems has received more and more attention in China. Bus transit plays a critical role in the whole urban traffic system due to its large capacity; thus if the operation efficiency of bus transit is increased, the quality of the whole urban traffic system will rise to a new level $[1,2]$.

Bus priority strategy can be mainly classified into two kinds: active priority strategy and passive priority strategy. Active priority strategy can make response to the bus presence in real-time. The examples of this kind of strategy are green extension, red truncation, phase insertion, and phase rotation [3]. Passive priority strategy does not operate in realtime and is based on the regular movement of bus streams. With respect to active priority strategy, passive priority strategy has the advantages of less investment and easier operation. When the bus percentage in the urban arterial is large, passive priority strategies can always have a very significant effect [4].

In the previous studies of passive priority strategies, Khasnabis and Rudraraju (1997) found that optimization of bus headway can reduce the delay time along the bus route [5]. Skabardonis (2000) proposed a set of passive bus priority strategies by using TRANSYT-7F [6]. Zhang (2003) studied intersection phase design and timing optimal methods aiming at minimizing average delay per person at signalized intersection. As buses have larger capacity than ordinary cars, these methods can have a promotion on bus priority [7]. $\mathrm{Ma}$ and Yang (2007) depicted the relationship between the departure frequency of a bus line, cycle length of signalized intersection, and the number of different signal status and found that the number of different signal states can affect the average bus delay [8].

Another kind of passive bus priority strategy is bus coordination. Coordination is an effective way to improve the overall efficiency of traffic along the arterial that cannot be achieved by single traffic signal control. There are two commonly used objectives of coordination. One is to provide maximum bandwidth for vehicles along the arterial, and the other is to minimize total delays, number of stops, or queue length of the arterial system $[9,10]$. As for bus coordination, it refers to making coordination schemes mainly according to bus operating parameters. However, there are not many researches in this respect. Estrada et al. (2009) provide a simulation methodology for minimizing the bus travel time by modifying the relative offset of green phase at the intersections [11]. 
Moreover, not much of the previous literature of passive bus priority has specially considered the factor of multiple bus lines on urban arterial. In fact, it is common to set multiple bus lines on urban arterials in China. Every bus line may have different operation characteristics. For example, a big-station bus has fewer stopping spots and thus costs shorter travel time on the arterial. This will increase the difficulty and complexity of bus passive priority control. Also, most of the previous literature about passive bus priority uses simulation methods. Analytical models have not yet received much attention.

With these reasons, this paper will focus on coordination of multiple bus lines on urban arterial. The primary objective of this paper is to reduce bus delay time and raise the operation efficiency of all bus lines on urban arterial. The main contents of this paper are (1) to define and analyze bus travel time on road section with the effect of multiple bus lines, (2) to formulate an analytical model to calculate total red time a bus encounters when travelling along the arterial according to bus travel time of each road section and timing parameters of each traffic signal, and (3) to optimize the offset scheme of traffic signals to minimize the total red time that all bus lines encounter in two directions of the arterial by using genetic algorithm.

\section{Data Site}

The data used in this paper is collected from Zhongshan North Street in the downtown area of Nanjing. Zhongshan North Street is a northwest (or southeast) direction arterial that provides a shorter route between urban area and suburban area in Nanjing; thus it is in an important geographical position. This paper selects a major part of Zhongshan North Street as the object arterial of this study which contains 11 traffic signals, including signalized intersections and signalized crosswalks. There are totally 7 bus lines operating through the object arterial: Line 16, Line 31, Line 34, Line 100, Line 151, Line 168, and Line $\mathrm{d} 2$. The structure, timing parameters of each signal, and other information of the object arterial are shown in Figure 1.

\section{Bus Travel Time on Road Section}

3.1. Definition of Bus Travel Time on Road Section. To be convenient, "section" is used to call the road space between two adjacent traffic signals. Bus travel time on road section in this paper is defined as follows: the time a bus consumed from the end of vehicle queue at the upstream traffic signal to the end of vehicle queue at the adjacent downstream traffic signal (not counting the red time at the upstream signal), as illustrated in Figure 2. This definition is based on the assumption that the upstream signal is unsaturated and it describes the common situation that buses share roadway with other vehicles. It is also applicable to the situation when there is a bus exclusive lane or bus-only approach simply by replacing vehicle queue with bus queue.

It can be seen from Figure 2 and the definition that bus travel time on road section is comprised of three parts: (1) queue clearance time at upstream traffic signal, including the propagation time of backward shock wave when the signal turns green and the time a bus moves forward following other vehicles, (2) running time on the section, and (3) bus dwell time (if there are bus stops on the section).

3.2. Analysis of Bus Travel Time on Road Section. The data of bus travel time collected from Zhongshan North Street is listed in Table 1. Bus stops included by each bus line are listed in Table 2. In Table 1, "sec $i$ " refers to the road space between traffic signal $i$ and traffic signal $i+1(i=1,2, \ldots 10)$. The data is collected through on-board investigation between 14:30 to 16:00 on Tuesday, Wednesday, and Thursday because traffic characteristics in urban streets are similar in these three weekdays. Also during the investigation period, all the intersections and crosswalks in Zhongshan North Street are unsaturated. The peak hours are improper for bus coordination because some of the intersections on Zhongshan North Street are saturated or oversaturated, such as Shanxi Road and Xinmofan Road. Therefore peak hours are improper for bus coordination and not chosen in this study.

It can be noticed from Table 1 that bus travel time on each road section varies from one bus line to another. The reasons lie in these aspects: (1) passenger demands varying from one bus line to another will lead to different bus dwell time; (2) different vehicle conditions of each bus line will lead to different running time; (3) different number of bus stops included by each bus line will lead to different total bus dwell time (as shown in Table 2). For example, Line d2 is a bigstation bus line and only stops at Sanpailou and Xiliuwan Park on the object arterial, while Line 31 has nearly all the bus stops listed in Table 2.

Another fact that can be noticed from Table 1 is that travel time of a bus line on each section varies from one direction to another. That's because buses share roadway with other vehicles on the object arterial; thus the imbalance of traffic volume in two directions will affect bus travel time. Also, the asymmetric layout of bus stops will also cause directional difference of bus travel time on the section. For example, as shown in Figure 1, Shanxi Road Stop in the direction from southeast to northwest is set on sec 3 while the opposite stop is set on $\sec 2$.

\section{Bus Time Calculation Model at Traffic Signal}

In this paper, bus time at traffic signal refers to the red time a bus encounters when arriving at the end of vehicle queue at this signal. During the red interval, a bus has to wait until the green signal starts and thus delays will occur. The amount of red time that a bus consumes at a signal is related to the signal timing parameters (including cycle length, green phase time, and the offsets along the arterial) and total travel time prior to this signal.

As for the coordination, cycle length of each traffic signal on the arterial needs to be the same or have multiple relationships at least to ensure the unique offset. In this paper, offset of signal $i$ refers to the difference of green start time between signal $i$ and the first signal $(i=1,2, \ldots 11)$. There 

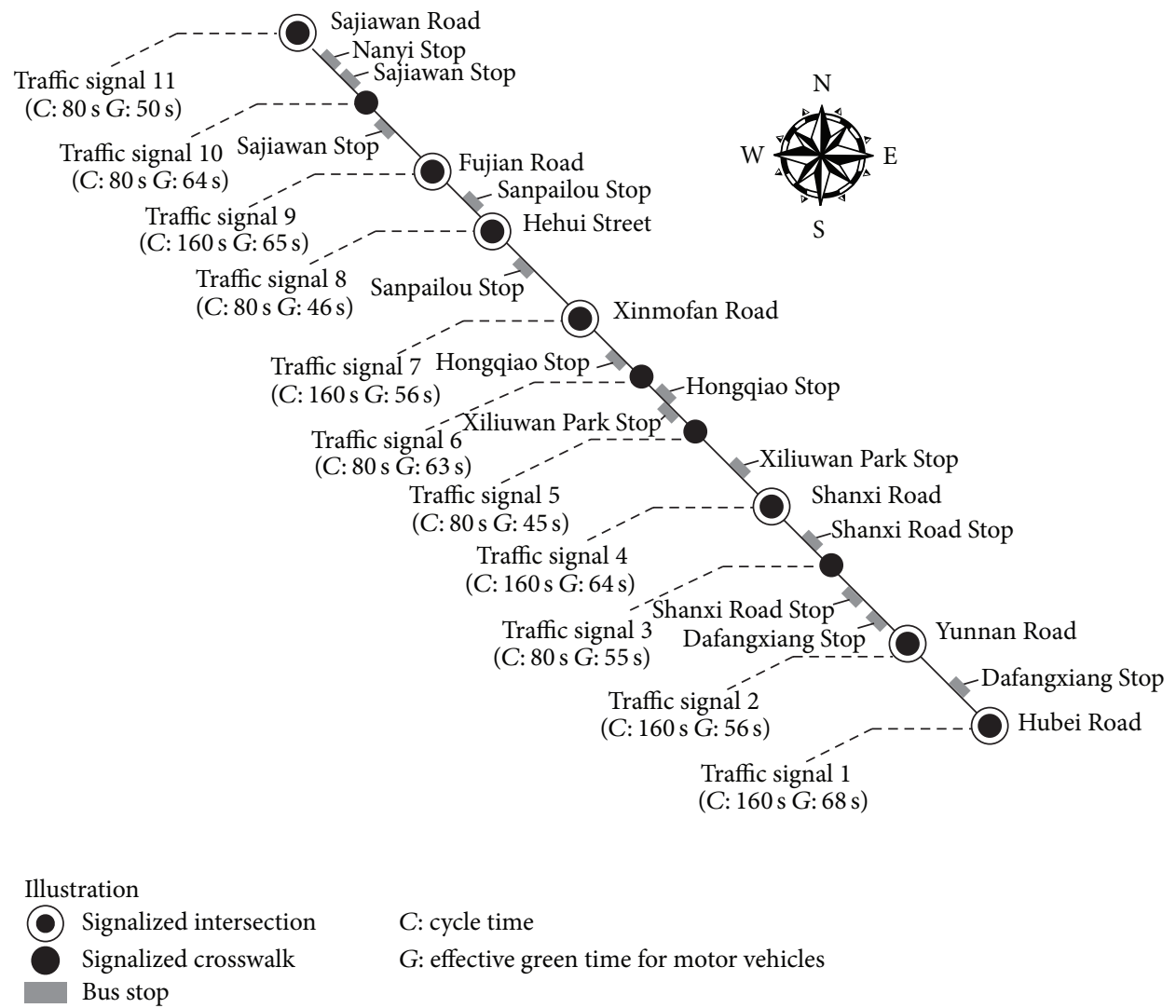

FIGURE 1: Information about the object arterial.
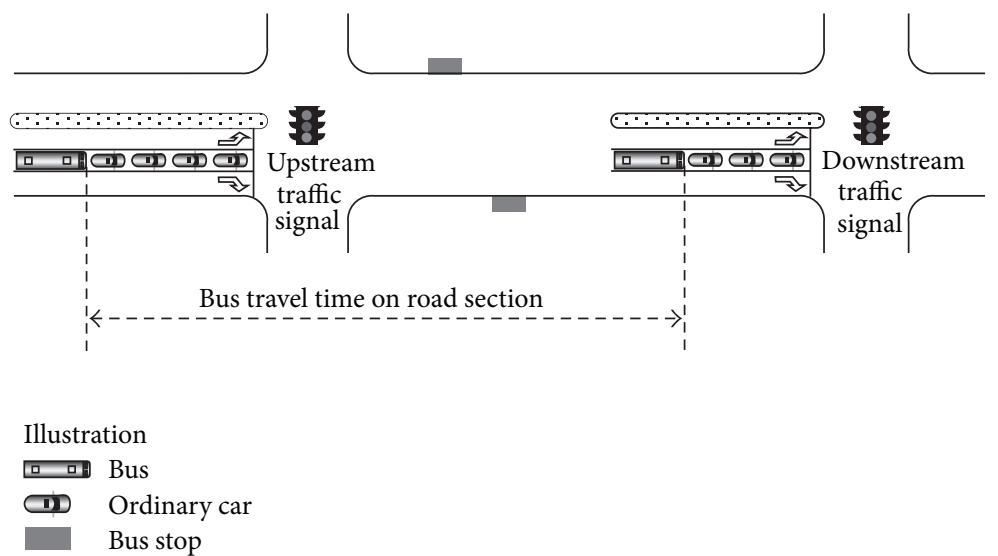

FIGURE 2: Illustration of bus travel time on road section.

are mainly two kinds of coordination: delay (or number of stops, maximal queue length) minimization and bandwidth maximization as mentioned in the previous paragraphs. In this paper, it is hard to provide bandwidth for every bus line due to the variety of bus travel time (as shown in Table 1). Therefore, delay minimization method is used. A model is formulated in the following paragraphs to calculate the total red time a bus encounters when travelling along the arterial according to bus travel time on each road section and timing plan of each traffic signal.
Figure 3 illustrates the travelling track of a bus in one direction. Timing plan and offset of each traffic signal are also displayed for red time calculation. Cumulative time $\mathrm{CT}_{i}$ is used to denote the total travel time prior to signal $i$, including total bus travel time on previous road section and total red time at previous signals. Thus cumulative time $\mathrm{CT}_{i}$ can be calculated as follows $\left(\mathrm{CT}_{1}=0\right)$ :

$$
\mathrm{CT}_{i}=\sum_{k=1}^{i-1}\left(T_{k}+r_{k}\right) \text {, }
$$


TABLE 1: Bus travel time of all the bus lines on each section.

\begin{tabular}{|c|c|c|c|c|c|c|c|c|c|c|}
\hline \multirow{2}{*}{ Bus line } & \multicolumn{10}{|c|}{ Bus travel time(s) (from southeast to northwest) } \\
\hline & $\operatorname{Sec} 1$ & $\operatorname{Sec} 2$ & $\operatorname{Sec} 3$ & $\operatorname{Sec} 4$ & $\operatorname{Sec} 5$ & $\operatorname{Sec} 6$ & $\operatorname{Sec} 7$ & $\operatorname{Sec} 8$ & $\operatorname{Sec} 9$ & $\operatorname{Sec} 10$ \\
\hline Line 16 & 69 & 39 & 63 & 73 & 60 & 20 & 99 & 74 & 37 & 57 \\
\hline Line 31 & 62 & 36 & 53 & 90 & 55 & 20 & 110 & 60 & 38 & 80 \\
\hline Line 34 & 105 & 46 & 69 & 95 & 66 & 28 & 97 & 77 & 33 & 49 \\
\hline Line 100 & 76 & 51 & 65 & 40 & 51 & 23 & 60 & 69 & 31 & 69 \\
\hline Line 151 & 72 & 49 & 72 & 62 & 57 & 17 & 66 & 86 & 43 & 64 \\
\hline Line 168 & 61 & 38 & 60 & 19 & 66 & 28 & 84 & 73 & 37 & 84 \\
\hline Line d2 & 48 & 41 & 42 & 49 & 46 & 19 & 72 & 60 & 32 & 41 \\
\hline \multirow{2}{*}{ Bus line } & \multicolumn{10}{|c|}{ Bus travel time(s) (from northwest to southeast) } \\
\hline & Sec 1 & $\operatorname{Sec} 2$ & $\operatorname{Sec} 3$ & $\operatorname{Sec} 4$ & Sec 5 & Sec 6 & $\operatorname{Sec} 7$ & Sec 8 & Sec 9 & Sec 10 \\
\hline Line 16 & 58 & 63 & 35 & 29 & 110 & 64 & 89 & 51 & 45 & 39 \\
\hline Line 31 & 77 & 107 & 67 & 31 & 207 & 118 & 116 & 41 & 65 & 40 \\
\hline Line 34 & 108 & 57 & 34 & 26 & 67 & 56 & 78 & 26 & 51 & 33 \\
\hline Line 100 & 92 & 151 & 22 & 24 & 43 & 86 & 103 & 42 & 63 & 64 \\
\hline Line 151 & 52 & 69 & 51 & 34 & 99 & 62 & 63 & 38 & 34 & 36 \\
\hline Line 168 & 39 & 85 & 60 & 30 & 95 & 62 & 107 & 59 & 62 & 31 \\
\hline Line d2 & 44 & 55 & 66 & 22 & 57 & 21 & 69 & 45 & 22 & 33 \\
\hline
\end{tabular}

TABle 2: Bus stops included by each bus line.

\begin{tabular}{|c|c|c|c|c|c|c|c|}
\hline \multirow{2}{*}{ Bus line } & \multicolumn{7}{|c|}{ Name of bus stops (from southeast to northwest) } \\
\hline & Dangfangxiang & Shanxi Road & Xiliuwan Park & Hongqiao & Sanpailou & Sajiawan & Nanyi \\
\hline Line 16 & $r$ & - & $r$ & $\checkmark$ & $\checkmark$ & $\checkmark$ & - \\
\hline Line 31 & $\checkmark$ & $\checkmark$ & $\checkmark$ & $\checkmark$ & $\checkmark$ & $\checkmark$ & $\checkmark$ \\
\hline Line 34 & $\checkmark$ & $\checkmark$ & $\checkmark$ & $\checkmark$ & $\checkmark$ & $\checkmark$ & - \\
\hline Line 100 & $\checkmark$ & $\checkmark$ & - & $\checkmark$ & $\checkmark$ & $\checkmark$ & $\checkmark$ \\
\hline Line 151 & $\checkmark$ & $\checkmark$ & $\checkmark$ & $\checkmark$ & $\checkmark$ & $\checkmark$ & - \\
\hline Line 168 & $\checkmark$ & - & $\checkmark$ & $\checkmark$ & $\checkmark$ & $\checkmark$ & $\checkmark$ \\
\hline Line d2 & - & - & $\checkmark$ & - & $\checkmark$ & - & - \\
\hline \multirow{2}{*}{ Bus line } & \multicolumn{7}{|c|}{ Name of bus stops (from northwest to southeast) } \\
\hline & Dangfangxiang & Shanxi Road & Xiliuwan Park & Hongqiao & Sanpailou & Sajiawan & Nanyi \\
\hline Line 16 & $r$ & - & $r$ & $\checkmark$ & $r$ & $\checkmark$ & - \\
\hline Line 31 & $\checkmark$ & - & $\checkmark$ & $\checkmark$ & $r$ & $\checkmark$ & - \\
\hline Line 34 & $r$ & $r$ & $r$ & $r$ & $r$ & $r$ & - \\
\hline Line 100 & $\checkmark$ & $\checkmark$ & - & $\checkmark$ & $r$ & $\checkmark$ & - \\
\hline Line 151 & $r$ & - & $r$ & $r$ & $r$ & $\checkmark$ & - \\
\hline Line 168 & $r$ & - & $\checkmark$ & $\checkmark$ & $r$ & $\checkmark$ & - \\
\hline Line d2 & - & - & $r$ & - & $r$ & - & - \\
\hline
\end{tabular}

where $T_{k}$ refers to the bus travel time on previous section $k$ and $r_{k}$ refers to the red time a bus encounters at previous signal $k$.

To calculate $r_{i}$, a nonnegative integer $n$ needs to be found to satisfy

$$
\mathrm{O}_{i}+(n-1) * C_{i}<\mathrm{CT}_{i}+t-t_{0} \leq \mathrm{O}_{i}+n * C_{i},
$$

where $C_{i}$ is the cycle length of signal $i, O_{i}$ is the offset of signal $i\left(0 \leq O_{i}<C_{i}\right), t_{0}$ is the green starting time of signal 1 in the travelling direction of the bus, and $t$ is the bus arrival time at signal $1\left(0 \leq t-t_{0}<C_{1}\right)$. Then $r_{i}$ can be calculated as

$$
r_{i}=\left\{\begin{array}{c}
O_{i}+n * C_{i}-\mathrm{CT}_{i}+t-t_{0} \\
0 \leq \mathrm{O}_{i}+n * C_{i}-\mathrm{CT}_{i}+t-t_{0} \leq C_{i}-G_{i} \\
0 \\
C_{i}-G_{i}<O_{i}+n * C_{i}-\mathrm{CT}_{i}+t-t_{0}<C_{i},
\end{array}\right.
$$

where $G_{i}$ is the effective green time of signal $i$. 


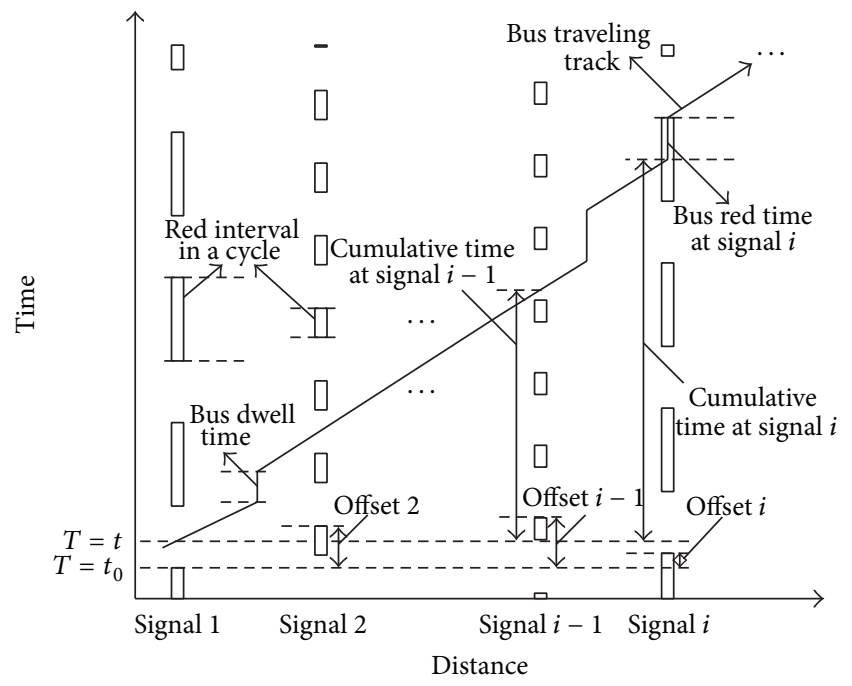

FIGURE 3: Illustration of bus time calculation model at traffic signal.

It can be seen that the calculation of red time a bus encounters at each signal is such an process: red time $r_{1}$ is first used to calculate the cumulative time $\mathrm{CT}_{2}$; then the red time $r_{2}$ at signal 2 can be calculated according to $\mathrm{CT}_{2}$, and so forth. The total red time $R$ of a bus along the arterial can be expressed as

$$
R=\sum_{i} r_{i}
$$

The total red time $R$ of a bus will change with bus arrival time $t$ at the first signal; therefore $R$ can be expressed as a function of $t$; that is, $R=R(t)$. Suppose the bus arrives with equal probability at any time at the first signal; then the average total red time $\bar{R}$ of a bus along the arterial can be calculated as

$$
\bar{R}=\frac{\left(\sum_{i=0}^{C_{1}} R\left(t_{i}\right)\right)}{C_{1}} .
$$

When adding the factors of bus driving direction and multiple bus lines, the total red time calculation model can be expressed as

$$
R_{T}=\sum_{i=1}^{N_{b}}\left(\bar{R}_{i, \text { outbound }}+\bar{R}_{i, \text { inbound }}\right)
$$

where $R_{T}$ is the total red time of all bus lines in two directions (outbound and inbound) of the arterial, $N_{b}$ is the total number of bus lines. It can be seen from (1) (6) that when the bus travel time on each road section and the timing plan of each traffic signal are determined, $R_{T}$ is only the function of offset scheme of signals along the arterial.

\section{Genetic Algorithm}

5.1. Advantages of Genetic Algorithm. Genetic algorithm (GA) as an intelligent optimization method has already been widely used in the area of traffic control. In this paper, GA is used to optimize the offset scheme of traffic signals along the arterial to achieve minimum total red time $R_{T}$ for all bus lines. Compared to traditional optimization algorithms, GA has the following advantages:

(1) GA does not require the solving model to be explicitly expressed. It only needs the fitness function and related variables. For the problem this paper studies, it is hard to get the explicit expression of total red time $R_{T}$. Thus the optimal value of $R_{T}^{*}$ cannot be easily obtained by traditional optimization methods. However, the only factor that will affect this total red time is the offset scheme of signals along the arterial. Therefore the problem of this paper can be solved by GA.

(2) The complexity of GA is approximately linearly correlated with the scale of the problem so that it will not cause the dimension disaster. The problem of this paper contains 11 traffic signals and the combination quantity of the offsets is large. Therefore GA is very suitable for solving this problem.

In the previous research of $\mathrm{GA}$ in the area of traffic control, Chang and Peng (2003) analyzed the real departurearrival model of traffic flow at intersection and developed a new method for urban arterial coordinate control based on genetic algorithm [12]. Singh et al. (2009) developed a traffic signal control strategy by using genetic algorithms which can provide optimum green time extensions and optimize signal timings in real time [13]. Yu et al. (2010) put forward a bilevel programming model in which passenger assignment model is the lower level and bus frequency optimization model is the upper level and then use genetic algorithm mainly to obtain the optimal bus frequencies to minimize the total travel time of passengers [14].

5.2. Algorithm Design. Generally speaking, the design of GA can be divided into five steps: chromosome coding, determination of fitness function, determination of select strategy, determination of genetic operator, and selection of the control parameters. First the initial population is generated through the chromosome coding of decision variables. Then proper select strategy is used to choose elite individuals in the current population according to their fitness. These elite ones will be the parents of the next generation. After that, genetic operators are applied to the parents to create the next generation. This process will continue until the terminal conditions are met.

(1) Chromosome Coding. Eight-bit binary coded string is used to denote the offset of each traffic signal shown in Figure 1 (except the first signal). This encoding method fits the problem of this paper because 8-bit binary coded string can denote every integer between 0 and 256, and the biggest cycle length is only $160 \mathrm{~s}$. The binary string of each signal is combined into a chromosome, and then the initial population is generated. 
TABLE 3: Distribution of optimal objective function values and the benefits.

\begin{tabular}{|c|c|c|c|c|c|c|}
\hline Range & $1180 \sim 1200$ & $1201 \sim 1250$ & $1251 \sim 1300$ & $1301 \sim 1350$ & $1351 \sim 1400$ & $1401 \sim 1500$ \\
\hline Occurrence number & 8 & 21 & 14 & 8 & 4 & 5 \\
\hline \multicolumn{4}{|c|}{ Minimum optimal value of objective function among the outputs } & \multicolumn{3}{|c|}{1180} \\
\hline \multicolumn{4}{|c|}{ Average objective function value under random offsets } & \multicolumn{3}{|c|}{1734} \\
\hline \multicolumn{4}{|c|}{ Reduced rate } & \multicolumn{3}{|c|}{$31.9 \%$} \\
\hline
\end{tabular}

A chromosome is also called genotype individual. Each genotype individual of every generation can be expressed as

$$
\begin{gathered}
x_{l}^{k}=\left[\left(b_{2,1}, b_{2,2}, \ldots, b_{2,8}\right), \ldots,\left(\ldots, b_{i, j}, \ldots\right), \ldots,\right. \\
\left.\left(b_{n, 1}, b_{n, 2}, \ldots, b_{n, 8}\right)\right]
\end{gathered}
$$

where $x_{l}^{k}$ is genotype individual $l(l=1,2, \ldots N$, and $N$ refers to the population size $)$ in generation $k\left(k=1,2, \ldots N_{0}\right.$, and $N_{0}$ refers to the maximum number of generations), $b_{i j}$ is binary number of signal $i$ in position $j$, and $n$ is the total number of signals. To decode this genotype individual to phenotype, that is, offset, the following equations are used (when $i=2, \ldots 11$ ):

$$
O_{i}= \begin{cases}\operatorname{int}\left(\frac{160}{2^{8}} \cdot \sum_{j=1}^{8} b_{i, j} \cdot 2^{8-j}\right), & C_{i}=160 \\ \operatorname{int}\left(\frac{80}{2^{7}} \cdot \sum_{j=2}^{8} b_{i, j} \cdot 2^{8-j}\right), & C_{i}=80\end{cases}
$$

When $i=1, O_{1}=0$.

(2) Determination of Fitness Function. The minimization of total red time $R_{T}$ mentioned in the previous texture is chosen as the objective function. The fitness of each individual in the population is directly taken as the value of objective function; that is, $R_{T}=f\left(O_{1}, O_{2}, \ldots O_{n}\right)$.

(3) Determination of Select Strategy. Breeding pool selection is chosen as the select strategy. First, elite individuals whose fitness ranks the top $20 \%$ of every generation are selected to form a breeding pool (these elite individuals directly go into the next generation). Elite ones in the breeding pool are randomly extracted in pairs to generate new individuals through crossover or mutation until the population of the next generation reaches the upper limit.

(4) Determination of Genetic Operator. Crossover operator and mutation operator are chosen to generate new individuals. Crossover operator is a function that breaks up two of the genotype individuals into four and combines them in some new way. Mutation operator is a function that makes small and random changes to an existing genotype individual [15].

(5) Selection of the Control Parameters. The main parameters of the algorithm are chosen as follows: $N=100, N_{0}=100$, the probability of crossover $p_{c}=0.8$, the probability of mutation $p_{m}=0.2$, and elite proportion of every generation $p_{e}=0.2$. When the number of generations reaches $N_{0}$, the algorithm terminates.

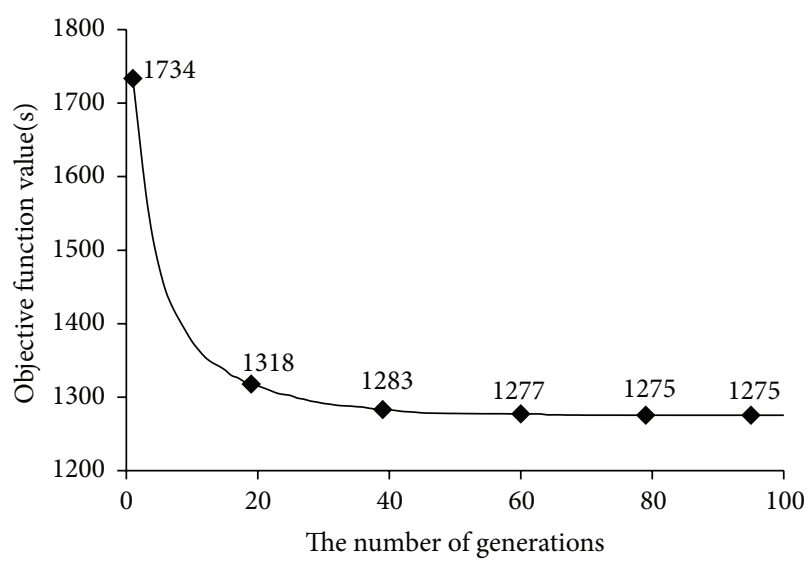

FIGURE 4: Average trend of objective function value.

5.3. Result of Genetic Algorithm. To solve the problem of coordination control for multiple bus lines in this paper, a procedure of GA has been developed with python 3.0 and runs for 60 times. The average trend of objective function value (total red time $R_{T}$ ) is shown in Figure 4 and the distribution of optimal objective function values is listed in Table 3.

Figure 4 illustrates that the algorithm has a fast convergence rate. The objective function value has already been close to the optimal value after the 20th generation of the population. Table 3 shows that it is difficult for the algorithm to find the exact globally optimal solution. The reason may lie in the large scale of problem in this paper. However, it does not prevent the algorithm from finding a satisfactory solution. The minimum of optimal objective function values among the outputs of the procedure is 1180 , and the corresponding offset scheme of traffic signals on the object arterial is $[0,82,29,0,63,40,136,43,142,15,41]$ (from signal 1 to signal 11 shown in Figure 1). Compared to the initial stage of optimization, when the offset of each signal along the arterial is randomly chosen, the average objective function value (i.e., total red time) is as high as 1734 . If the current offsets on Zhongshan North Street are randomly set, the total red time of all bus lines can be reduced by $31.9 \%$ as shown in Table 3 .

\section{Conclusions}

This paper analyzed the characteristic of bus travel time on road section with the effect of multiple bus lines and formulated a bus time model at traffic signals to calculate the total red time a bus encounters when travelling along the arterial and used genetic algorithm to optimize the offset 
scheme of traffic signals on the major part of Zhongshan North Street in the city of Nanjing. The optimization objective is to minimize the total red time of all the bus lines in two directions of the street. The results show that the model and algorithm can reduce the total red time by $31.9 \%$ on the arterial.

The methods in this paper only need to collect bus travel time and make a proper design of the offsets in the arterial, without having to change other signal timing parameters of intersections and crosswalks and thus can be easily operated and extensively applied. The model and algorithm can also be extended to solve the problem of bus coordination in the network if a few alterations are made. However, the stochastic characteristic of bus travel time on road section for each bus line is not considered in this paper due to lack of data. This work will be conducted in the future study.

\section{Conflict of Interests}

The authors declare that there is no conflict of interests regarding the publication of this paper.

\section{Acknowledgments}

This research is supported by National Natural Science Foundation of China under Grant no. 61374195. The authors acknowledge the assistance of colleagues in the data collection and paper editing process.

\section{References}

[1] C. D. Hunter, Guidelines for the successful implementation of transit signal priority on arterials [Ph.D. thesis], University of Washington, 2000.

[2] M. Vasudevan, Robust optimization model for bus priority under arterial progression [Ph.D. thesis], University of Maryland, 2005.

[3] W. Ekeila, T. Sayed, and M. E. Esawey, "Development of dynamic transit signal priority strategy," Transportation Research Record: Journal of the Transportation Research Board, vol. 2, no. 2111, pp. 1-9, 2009.

[4] W. Ma, Bus signal priority control theories based on exclusive bus lane [Doctoral dissertation], Tongji University, Shanghai, China, 2007.

[5] S. Khasnabis and R. K. Rudraraju, "Optimum bus headway for preemption: a simulation approach," Transportation Research Record: Journal of the Transportation Research Board, no. 1603, pp. 128-136, 1997.

[6] A. Skabardonis, "Control strategies for transit priority," Transportation Research Record: Journal of the Transportation Research Board, vol. 1727, pp. 20-26, 2000.

[7] W. Zhang, The study of the urban public transportation priority transit technology and estimate method [Ph.D. thesis], Southeast University, Nanjing, China, 2003.

[8] W. Ma and X. Yang, "A passive transit signal priority approach for bus rapid transit system," in Proceedings of the IEEE Intelligent Transportation Systems Conference (ITSC '07), pp. 413-418, Seattle, Wash, USA, October 2007.

[9] K. Lu, X. Zeng, L. Li, and J. Xu, "Two-way bandwidth maximization model with proration impact factor for unbalanced bandwidth demands," Journal of Transportation Engineering, vol. 138, no. 5, pp. 527-534, 2012.

[10] N. H. Gartner and C. Stamatiadis, "Arterial-based control of traffic flow in urban grid networks," Mathematical and Computer Modelling, vol. 35, no. 5-6, pp. 657-671, 2002.

[11] M. Estrada, C. Trapote, M. Roca-Riu, and F. Robusté, "Improving bus travel times with passive traffic signal coordination," Transportation Research Record: Journal of the Transportation Research Board, vol. 2111, pp. 68-75, 2009.

[12] Y. Chang and G. X. Peng, "Urban arterial road coordinate control based on genetic algorithm," Journal of Traffic and Transportation Engineering, vol. 3, no. 2, pp. 106-112, 2003.

[13] L. Singh, S. Tripathi, and H. Arora, “Time optimization for traffic signal control using genetic algorithm," International Journal of Recent Trends in Engineering, vol. 2, no. 2, pp. 4-6, 2009.

[14] B. Yu, Z. Z. Yang, and J. B. Yao, "Genetic algorithm for bus frequency optimization," Journal of Transportation Engineering, vol. 136, no. 6, pp. 576-583, 2010.

[15] T. Segaran, Programming Collective Intelligence: Building Smart Web 2.0 Applications, O’Reilly Media, Sebastopol, Calif, USA, 2007. 

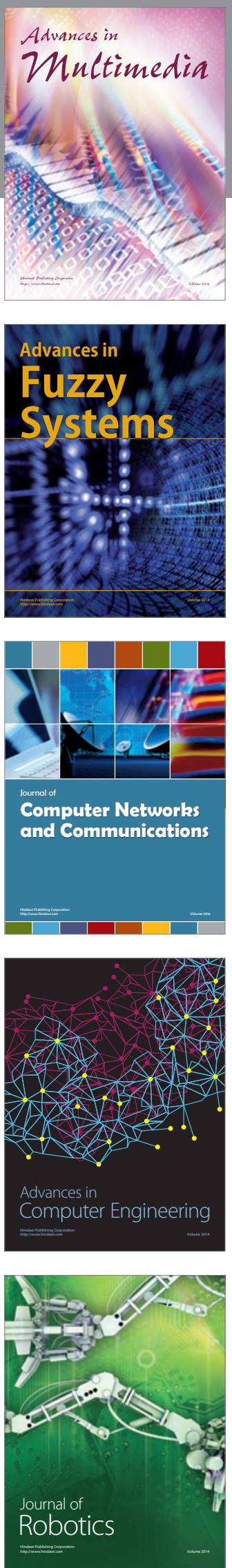

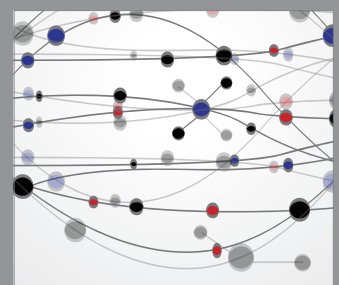

The Scientific World Journal
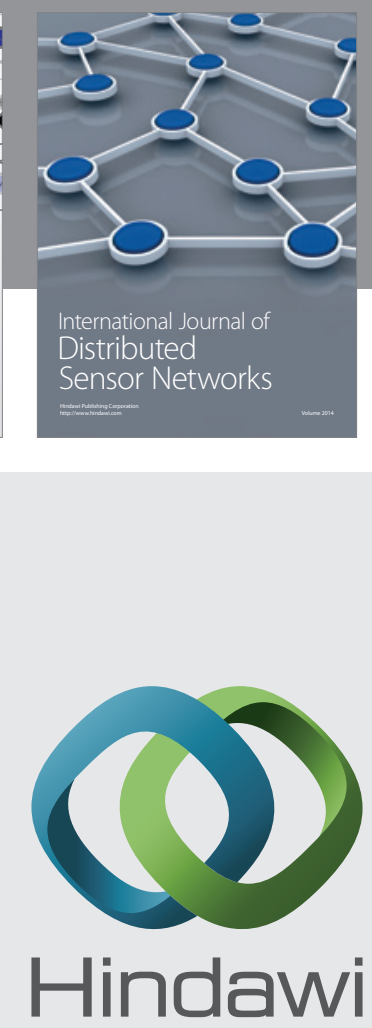

Submit your manuscripts at

http://www.hindawi.com
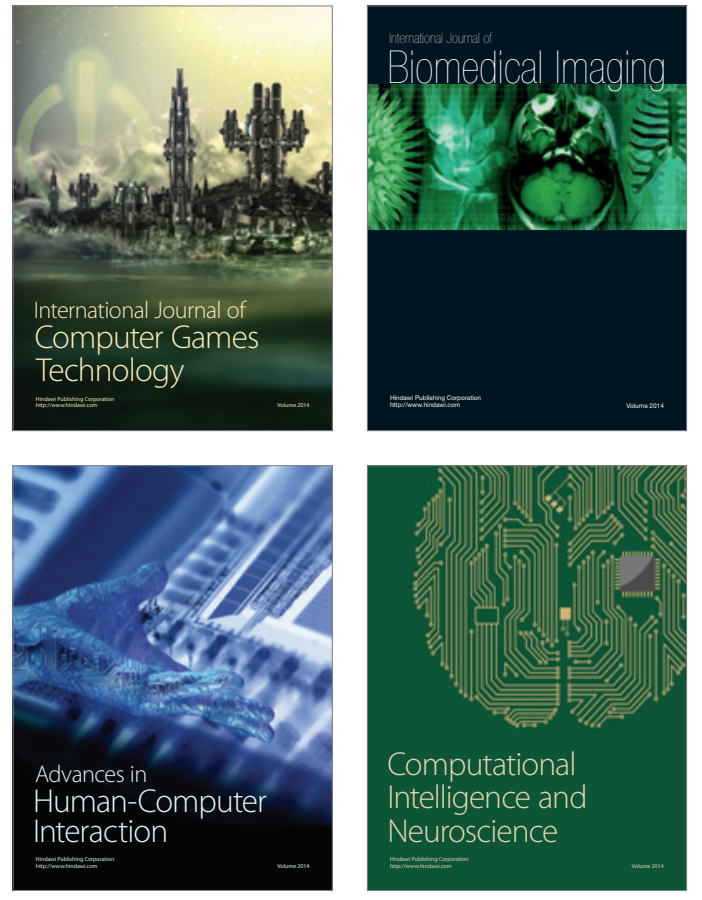
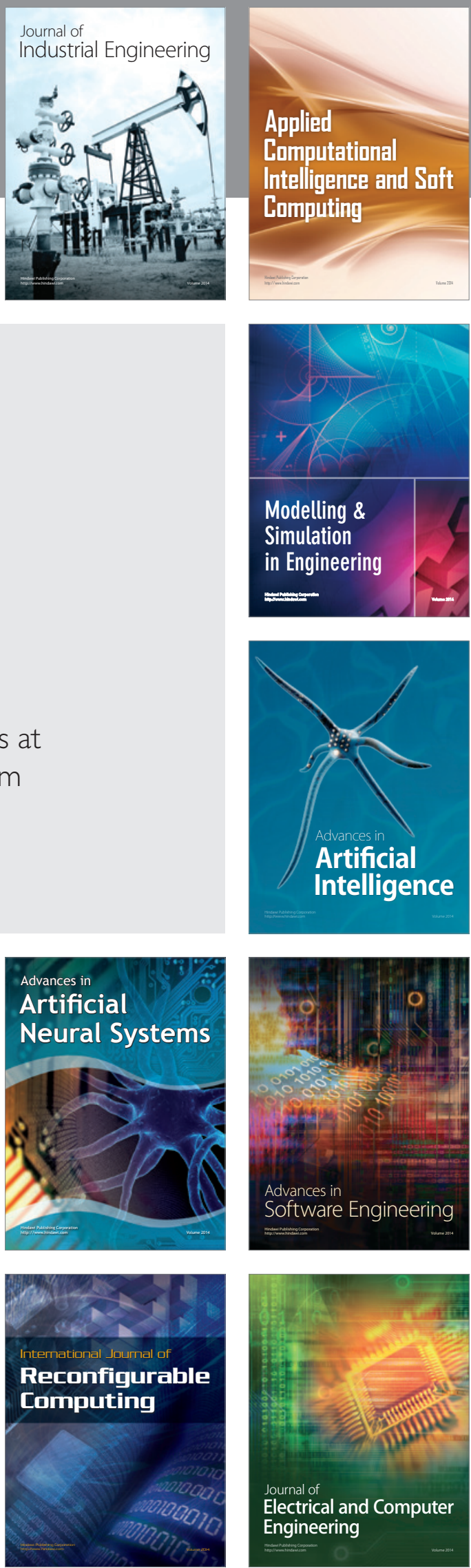